\title{
Upper Abdominal Resection for Isolated Metastatic Lesions in Recurrent Cervical Cancer
}

\author{
ALEXANDRU FILIPESCU ${ }^{1,2}$, IRINA BALESCU ${ }^{3}$ and NICOLAE BACALBASA ${ }^{1}$ \\ ${ }^{1}$ Department of Obstetrics and Gynecology, \\ Carol Davila University of Medicine and Pharmacy, Bucharest, Romania; \\ ${ }^{2}$ Department of Obstetrics and Gynecology, Elias Hospital, Bucharest, Romania; \\ ${ }^{3}$ Department of General Surgery, Ponderas Academic Hospital, Bucharest, Romania
}

\begin{abstract}
Upper abdominal involvement in recurrent cervical cancer usually occurs as part of systemic disease and is most often amenable to palliative chemotherapy. However, in certain cases in which oligometastatic disease is proven, surgery might be considered. We present a case series of six patients submitted to surgery for upper abdominal oligometastases with cervical origin. The main surgical procedures performed included atypical hepatectomy (in five cases) and splenectomy (in one case). In all cases, the cervical origin of the lesions was revealed by histopathological studies. After a median followup period of 1 year, there was no evidence of recurrent disease in five cases, while the sixth case was diagnosed with disseminated lesions and is under palliative chemotherapy. In conclusion, upper abdominal resection for oligometastatic disease from cervical cancer can improve the long-term survival in selected cases.
\end{abstract}

Cervical cancer remains a major health problem worldwide, a significant number of cases being diagnosed in advanced stages of the disease. Most often, neoplastic cells with cervical origin spread via local permeation process or lymphatic routes, leading to local pelvic recurrence or lymphatic metastasis. In certain cases, hematogenous spread leading to the appearance of distant lesions may occur; this represents the worst scenario, disseminated lesions most often being diagnosed. In this case, the patient can be only submitted to palliative chemotherapy, with very poor results in terms of overall survival. In very rare cases, isolated lesions can be found, making the patient a perfect candidate for surgery.

Correspondence to: Irina Balescu, Buzau, 2A Caraiman Street, Postal code 120143, Romania. Tel: +40 724077709, e-mail: irina.balescu@ponderas-ah.ro

Key Words: Cervical cancer, oligometastatic disease, resection.

\section{Patients and Methods}

We present a case series of six patients submitted to upper abdominal resections for oligometastatic lesions with cervical origin submitted to surgery between 2014 and 2016. Due to the fact that all lesions were diagnosed at more than 6 months from cervical cancer diagnosis, they were all considered as metachronous lesions. In all cases, postoperative complications were classified according to Dindo-Clavien scale (1). All patients had been initially submitted to surgery at the Fundeni Clinical Hospital and Ilfov County Hospital for both initial and metastatic disease. Exclusion criteria consisted of presenting disseminated lesions at the time of surgery. The endpoint of the study was to assess the long-term outcomes after upper abdominal resection for isolated metachronous lesions from cervical cancer.

\section{Results}

Five out of the six patients included in the current study were diagnosed with liver metastases from cervical cancer, the mean disease-free survival being 14 months. The mean age at the diagnosis of the hepatic lesions was 54.4 years (range $=43-59$ years). Regarding the initial stage at diagnosis, two cases were diagnosed with International Federation of Obstetrics and Gynecology (FIGO) stage IIB cervical cancer, two with FIGO stage IIIA tumor, while the fifth patient was diagnosed with a FIGO stage IVA lesion. At that time, surgery consisted of total radical hysterectomy with bilateral adnexectomy in four cases and anterior pelvic exenteration in the fifth case; all the cases also underwent pelvic bilateral and para-aortic lymph node dissection. At the time of recurrence, all cases were diagnosed with unique hepatic lesions, the median size of the liver tumor being $3 \mathrm{~cm}$ (range $=1-6 \mathrm{~cm}$ ). In one case that had been initially diagnosed with a FIGO stage IIIA tumor, the hepatic lesion was associated with the presence of a pelvic recurrence.

Three out of the four cases were submitted to atypical hepatectomies (Figures 1-4), while the fourth case necessitated a segmental resection involving segments $\mathrm{V}$ and 
VI according to Couinaud's classification (Figure 5). One of the four cases which benefitted from a minor liver resection was also submitted to total pelvic exenteration. The patient had been initially diagnosed with a stage IIIA tumor and developed pelvic and hepatic recurrence 10 months after the initial diagnosis. Although she was submitted to surgery with curative intent, both at the time of initial diagnosis and at the time of the diagnosis of the recurrent tumor, she developed disseminated recurrences 9 months after the second surgery and is currently under palliative chemotherapy.

Postoperatively, a single patient (the one submitted to segmental resection) developed a biliary fistula which was successfully managed in a conservative manner.

The sixth patient, who was submitted to splenectomy for isolated uterine cervix splenic metastasis, had been previously diagnosed with a FIGO stage IIB cervical tumor at the age of 30 years; at that time she had been submitted to neoadjuvant radiochemotherapy followed by total radical hysterectomy en bloc with bilateral adnexectomy and pelvic lymph node dissection. Eighteen months later, she was diagnosed with a solitary splenic tumor and she underwent splenectomy; the histopathological studies confirmed it to be of uterine cervix origin. Eighteen months later, she remains free of any recurrent disease (Figure 6).

Intraoperative aspects demonstrating the effectiveness of surgery for isolated upper abdominal metastases from cervical cancer are presented in Figures 1-6.

\section{Discussion}

The development of hematogenous metastases in cervical cancer is not totally understood. However, it seems to be an independent process, which is not influenced by the lymphatic pattern of spread (the presence of metastatic and micrometastatic lymphatic tumoral foci having no influence on the risk of hematogenous spread) (2-4).

One of the most relevant studies conducted on this theme was published in 2003 by a German and Austrian team; this study demonstrated that the risk of distant metastases in cervical cancer is related to the presence of isolated tumor cells in the bone marrow of these patients at the time of primary diagnosis; this finding seems to be strongly correlated with a higher risk of distant metastases at a certain moment in the evolution of the disease. According to these authors, the presence of isolated tumor cells in the bone marrow of patients with cervical cancer is not correlated with FIGO stage at diagnosis, tumor stage, pelvic or para-aortic lymph node metastasis, histopathological type or grading, or blood vessel invasion, nor with the menopausal status of the patient. However, once the presence of metastatic tumor cells was revealed, the risk of distant metastasis significantly increased. Moreover, the presence of such tumoral islets seems to be a strong predictor for a shorter disease-free survival interval; in consequence, the authors underlined the fact that administration of systemic therapy in these cases might significantly reduce the rate of appearance of distant lesions (4).

When it comes to the most common sites of hematogenous spread in cervical cancer, these are represented by the lungs, bone and liver $(5,6)$. For liver metastases with gynecological origin, fewer than $10 \%$ could be suitable for surgery although it is estimated that up to half of patients diagnosed with gynecological malignancies will be diagnosed with hepatic lesions at autopsy studies (7-9). Studies conducted on large series of patients with noncolorectal non-neuroendocrine liver metastases demonstrated that hepatic resection is associated with a maximal benefit in terms of survival in cases diagnosed with solitary lesions, in the absence of extrahepatic disease, when optimal cytoreduction is expected and if the disease-free survival period is longer than 12 months. These conditions also seem to have a strong influence on the outcomes of patients diagnosed with cervical cancer liver metastases (9-11). The first case-series of liver resection for cervical cancer liver metastases was published five decades ago by Brunschwig (12); he reported 24 cases of hepatic resection for metastatic carcinoma, four of them being diagnosed with endometrial and uterine cervix origin. Although at that time the benefit in terms of survival was limited (three cases dying during the early postoperative period while the fourth case experienced 18-month survival), the improvement of surgical techniques and postoperative management contributed to lower perioperative mortality. Due to these aspects, liver surgery has been successfully included as part of the therapeutic armamentarium for isolated liver metastases from cervical cancer.

One of the most recent studies regarding the benefits of liver resection for uterine cervix metastases was conducted by Bacalbasa et al. (13). The study was conducted at the Fundeni Clinical Institute, Bucharest, Romania, between 2002 and 2014, and included 15 patients diagnosed with liver metastases of carcinoma of the uterine cervix; among these cases, there were two patients with synchronous lesions and 13 with metachronous lesions. The median overall survival of patients submitted to hepatic resection for metachronous lesions was 18 months, being significantly influenced by the absence of extrahepatic disease and by the tumoral degree of differentiation. Another important conclusion of the article was that the type of resection (minor versus major hepatectomy) did not have an impact on the overall survival. For the two patients diagnosed with synchronous lesions, they reported survival after liver resection of 9 and 89 months, respectively. These results enabled the authors to consider that in selected cases, liver resection for isolated liver metastases can significantly affect the overall prognosis (13). 


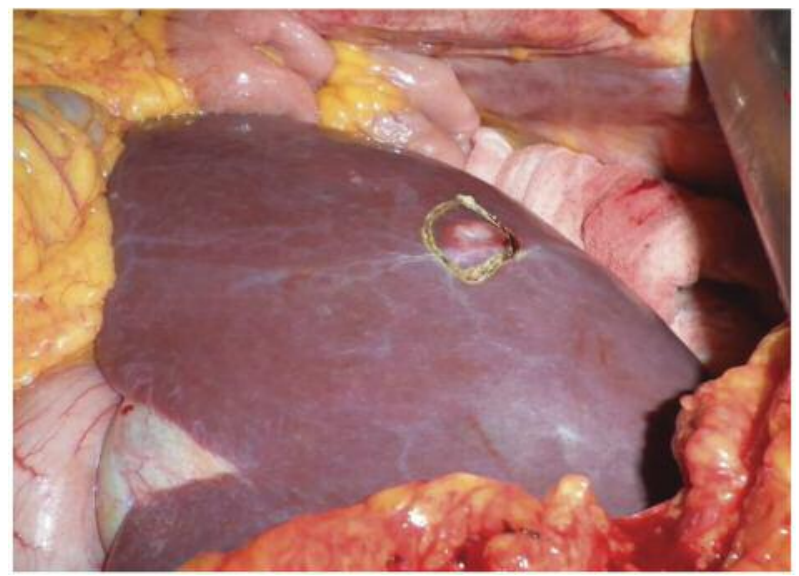

Figure 1. Delimitation of a $1.5 \mathrm{~cm}$ liver metastasis of cervical cancer in a patient who also presented a pelvic recurrence.

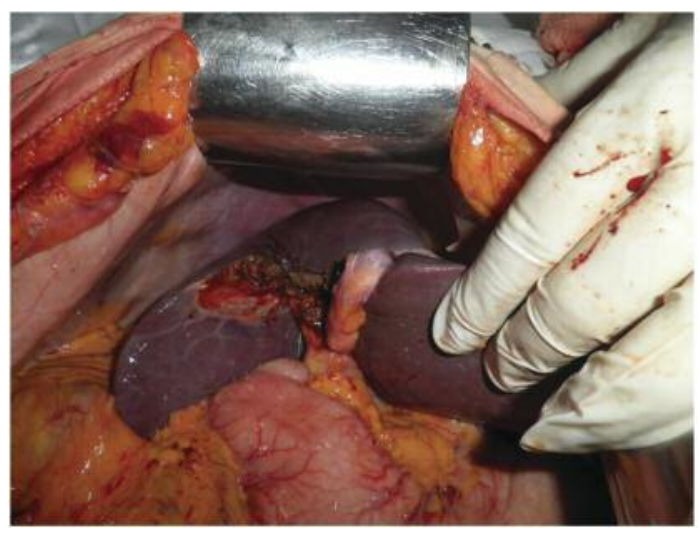

Figure 2. The final aspect after atypical hepatectomy for a $3 \mathrm{~cm}$ cervical cancer liver metastasis.

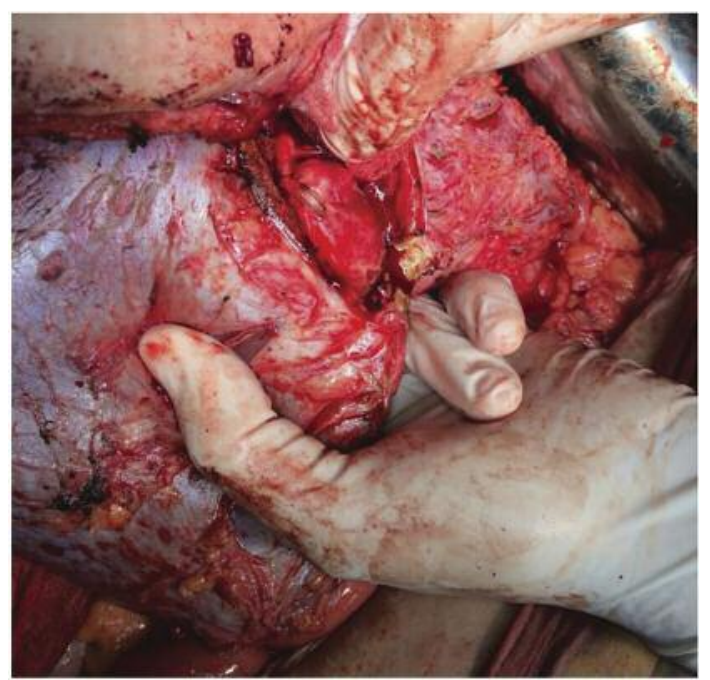

Figure 3. Mobilization of a $3 \mathrm{~cm}$ cervical cancer liver metastasis.

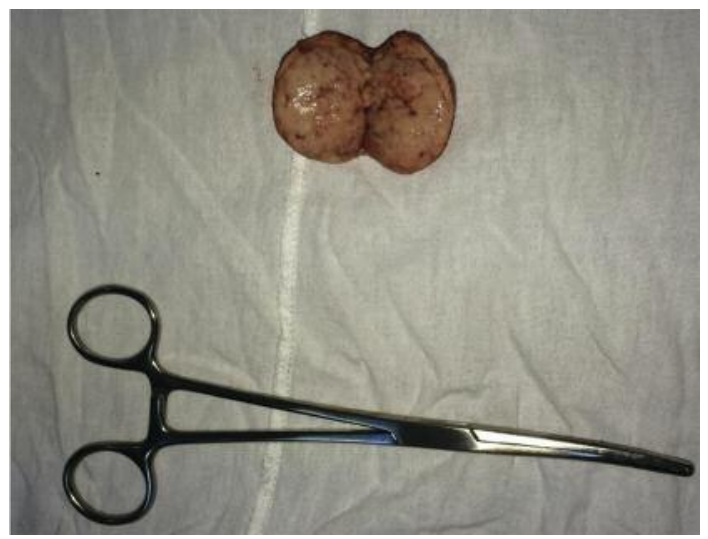

Figure 4. Gross view of specimen of liver metastasis with cervical cancer origin.

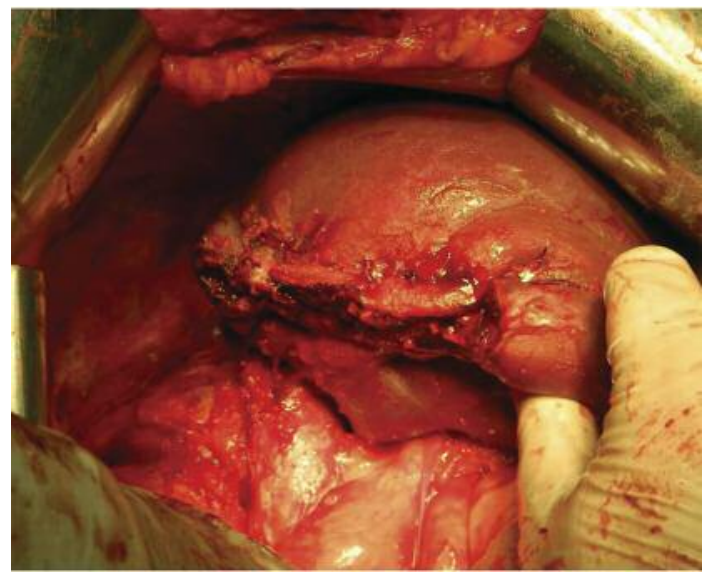

Figure 5. The aspect after segmental resection (segment VI) for a $5 \mathrm{~cm}$ liver metastasis with cervical origin.

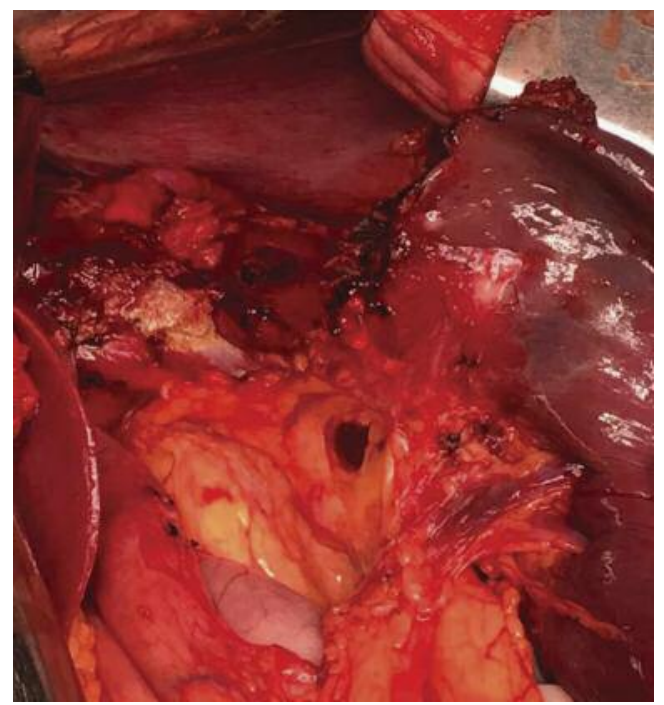

Figure 6. Splenic metastasis with cervical cancer origin . 
Regarding the incidence of splenic metastasis, autopsy studies reported that it ranges between 1.6 and 30\% (14); however, most cases are diagnosed with splenic lesions as part of systemic recurrence and therefore surgery is not indicated (15). The rarity of cases presenting with splenic metastases can be explained throughout multiple mechanisms, the main ones consisting of the tortuosity of the splenic artery as well as the lack of afferent lymphatic vessels at the level of the splenic parenchyma, rhythmic contractions of the spleen, the presence of the splenic capsule (acting as a barrier against neoplastic propagation) and of the possible anti-neoplastic properties of splenic tissue (16). Most often, splenic metastases as part of systemic disease originate from breast, lung, colorectal, ovarian carcinomas or melanomas, while isolated splenic metastases are more frequently encountered in colorectal and ovarian cancer (17). Solitary metastasis from cervical cancer was first reported by Brufman et al. in 1977 (18), only few cases being described since then (19-22). Although experience regarding such cases exists only from a few reported case reports, it seems that surgery might be successfully performed with radical intent. However, the fact that, although not visible, other microscopic disseminated lesions might be present, should not be overlooked, so the patients should be confined in the postoperative period to the oncologist; in this way the patient will benefit from the administration of adjuvant chemotherapy to treat the possible microscopic lesions and to consolidate the surgical procedure which had previously removed the macroscopic lesions. So far it seems that a combination of cisplatin and paclitaxel represents the most efficient regimen for these cases (19).

As can be seen from the data that have been reported so far, isolated upper abdominal metastases from cervical cancer is quite a rare situation. However, whenever it is encountered, it seems that an aggressive surgical approach represents the best therapeutic option in order to achieve good control of the disease. As demonstrated in the present study, both splenic and liver resections can be safely performed, with acceptable rates of perioperative morbidity. Therefore, surgery should become part of the therapeutic armamentarium in such cases. Moreover, it seems that this surgical approach can be safely performed even in the presence of other isolated lesions, one of the patients of the current study being submitted to both pelvic exenteration and liver resection in order to provide a potentially curative solution.

\section{Conclusion}

Although isolated, upper abdominal metastases from cervical cancer represent a rare situation, and experience regarding such cases usually originates from case reports or case series, it seems that radical surgery is the most efficient therapeutic option in order to obtain a good control of the disease and to improve survival. However, larger studies are still needed to establish which cases might benefit the most.

\section{References}

1 Dindo D, Demartines $\mathrm{N}$ and Clavien PA: Classification of surgical complications: a new proposal with evaluation in a cohort of 6336 patients and results of a survey. Ann Surg 240: 205-213, 2004.

2 Lindemann F, Schlimok G, Dirschedl P, Witte J and Riethmuller G: Prognostic significance of micrometastatic tumour cells in bone marrow of colorectal cancer patients. Lancet 340: 685-689, 1992.

3 Braun S, Cevatli BS, Assemi C, Janni W, Kentenich CR, Schindlbeck C, Rjosk D and Hepp F: Comparative analysis of micrometastasis to the bone marrow and lymph nodes of nodenegative breast cancer patients receiving no adjuvant therapy. $\mathrm{J}$ Clin Oncol 19: 1468-1475, 2001.

4 Janni W, Hepp F, Strobl B, Rack B, Rjosk D, Kentenich C, Schindlbeck C, Hantschmann P, Pantel K, Sommer H and Braun $\mathrm{S}$ : Patterns of disease recurrence influenced by hematogenous tumor cell dissemination in patients with cervical carcinoma of the uterus. Cancer 97: 405-411, 2003.

5 Carlson V, Delclos L and Fletcher GH: Distant metastases in squamous-cell carcinoma of the uterine cervix. Radiology 88 : 961-966, 1967.

6 Carvalho L, Azevedo I, Salgado L, Ferreira ES, Henrique R, de Carvalho RG and Vieira E: Squamous cell carcinoma of the cervix metastatic to the spleen - case report. Gynecol Oncol 67: 107-110, 1997.

7 Chi DS, Fong Y, Venkatraman ES and Barakat RR: Hepatic resection for metastatic gynecologic carcinomas. Gynecol Oncol 66: 45-51, 1997.

8 Rose PG, Piver MS, Tsukada Y and Lau TS: Metastatic patterns in histologic variants of ovarian cancer. An autopsy study. Cancer 64: 1508-1513, 1989.

9 Adam R, Chiche L, Aloia T, Elias D, Salmon R, Rivoire M, Jaeck D, Saric J, Le Treut YP, Belghiti J, Mantion G and Mentha $\mathrm{G}$ : Hepatic resection for noncolorectal nonendocrine liver metastases: analysis of 1,452 patients and development of a prognostic model. Ann Surg 244: 524-535, 2006.

10 Neumann UP, Fotopoulou C, Schmeding M, Thelen A, Papanikolaou G, Braicu EI, Neuhaus P and Sehouli J: Clinical outcome of patients with advanced ovarian cancer after resection of liver metastases. Anticancer Res 32: 4517-4521, 2012.

11 Ercolani G, Grazi GL, Ravaioli M, Ramacciato G, Cescon M, Varotti G, Del Gaudio M, Vetrone G and Pinna AD: The role of liver resections for noncolorectal, nonneuroendocrine metastases: experience with 142 observed cases. Ann Surg Oncol 12: 459-466, 2005.

12 Brunschwig A: Hepatic lobectomy for metastatic cancer. Cancer 16: 277-282, 1963.

13 Bacalbasa N, Balescu I, Dima S and Popescu I: Hepatic resection for liver metastases from cervical cancer is safe and may have survival benefit. Anticancer Res 36: 3023-3027, 2016.

14 Goktolga U, Dede M, Deveci G, Yenen MC, Deveci MS and Dilek S: Solitary splenic metastasis of squamous cell carcinoma of the uterine cervix: a case report and review of the literature. Eur J Gynaecol Oncol 25: 742-744, 2004. 
15 Taga S, Sawada M, Nagai A, Yamamoto D and Hayase R: Splenic metastasis of squamous cell carcinoma of the uterine cervix: a case report and review of the literature. Case Rep Obstet Gynecol 2014: 798948, 2014.

16 Koh YS, Kim JC and Cho CK: Splenectomy for solitary splenic metastasis of ovarian cancer. BMC Cancer 4: 96, 2004.

17 Comperat E, Bardier-Dupas A, Camparo P, Capron F and Charlotte F: Splenic metastases: clinicopathologic presentation, differential diagnosis, and pathogenesis. Arch Pathol Lab Med 131: 965-969, 2007.

18 Brufman G, Biran S, Goldschmidt Z and Freund U: Solitary metastatic involvement of the spleen in squamous cell carcinoma of the cervix. Harefuah 92: 349-350, 1977.

19 Dixit J, Mohammed N and Shetty P: Splenic metastasis from cancer of uterine cervix - a rare case. Indian J Surg Oncol 7: 479-483, 2016.
20 Sharma P, Chatterjee P and Mazumdar B: Recurrent carcinoma cervix presenting as metastatic splenomegaly: (18)F-FDG $\mathrm{PET} / \mathrm{CT}$ findings in a rare scenario. Indian J Nucl Med 29: 200201, 2014.

21 Bhardwaj S, Mahajan D and Vir Gupta Y: Metastatic squamous cell carcinoma of the cervix presenting as a splenic cyst. J K Science 10(3): 146-148, 2008.

22 Bacalbasa N, Balescu I, Marcu M, Oprescu DN and Anca AF: Solitary splenic metastasis after surgically-treated cervical cancer - a case report and literature review. Anticancer Res 37: 2615-2618, 2017.

Received November 21, 2017

Revised January 1, 2018

Accepted January 10, 2018 